\title{
A WBS-Based Plan Changeability Measurement Model for Reducing Software Project Change Risk
}

\author{
Sen-Tarng Lai
}

\begin{abstract}
In software development process, it is necessary to face challenge for plan changes. In order to effectively reduce development risk, software project must overcome the impact of plan change. In plan change process, affected development documents and plan items should be effectively isolated and immediately modified to reduce software development risk. In software project management plan (SPMP), Work Breakdown Structure (WBS) is a critical and necessary planning tool. For this, WBS-based plan must have high isolation capability and modification flexibility to reduce plan change risk. In this paper, in order to increase WBS-based plan change capability, changeability factors are discussed, and a WBS-based Plan Changeability Measurement (WBSPCM) model is proposed. Applied the quantification mechanism of WBSPCM model, WBS-based plan changeability problem and defect can be easily identified. Used rule-based correction and improvement manner, WBS-based plan can concretely increase changeability and reduce software development risk.
\end{abstract}

Index Terms-WBS, measurement model, changeability, software development risk.

\section{INTRODUCTION}

In software project development process, it is necessary to face challenge for plan changes. Software project must overcome the impact of plan changes to effectively reduce development risk [1], [2]. Some events, which include error correction, requirement revision, environment evolution, and resource adjustment possibly cause to plan changes. Any plan change always affects software development operation. Plan changes not only have to invest extra resource and cost, but also may cause project delay [1], [2]. In plan changes process, affected development documents and planned items unable to effectively isolate, will increase software development risk. In addition, affected development documents and planned items unable completely and correctly modify, will greatly reduce project success ratio. There are many factors may affect the software project failure. One of critical issues is software project management plan (SPMP) can not immediately revise and effectively adjust with the change events. For this, SPMP must have high isolation capability and modification flexibility to reduce plan changes risk.

The SPMP is drawn up when the specifications have been signed off by the client. There are many ways to construct an SPMP. One of the best is IEEE standard 1058 [3], [4]. IEEE 1058 SPMP standard defines project organization,

Manuscript received June 6, 2013; revised September 16, 2013.

Sen-Tarng Lai is with Department of Information Technology and Management, Shih Chien University, Taipei, Taiwan (e-mail: stlai@mail.usc.edu.tw). management process plans, technical process plans and supporting process plans four major items. In order to concretely and effectively accomplish requirement specifications, large scale and complicate project should be suitable decomposed. Work Breakdown Structure (WBS) is a critical and necessary planning item in SPMP [5]. Plan Change operations always may affect the WBS. For this, WBS-based plan should have high isolation capability and modification flexibility to increase changeability and reduce plan changes risk. WBS-based plan with important quality characteristics is a critical factor to enhance changeability for affected development documents and planned items. Therefore, how to increase WBS-based plan changeability is worthily studied issue for reducing software development risk.

The paper discusses and surveys WBS-based plan related characteristics and WBS-based plan changeability factors. In plan review activity, using inspection checklists help concretely collect WBS-based plan changeability quantified factors. Individual factor or measurement can only measure or evaluate the specific maintain quality characteristic [6]. In order to improve and enhance WBS-based plan changeability, individual factor or measurement should make appropriate combination. Based on the metric combination model, in this paper presents a WBS-based Plan Changeability Measurement (WBSPCM) model. In WBSPCM model, planned documents quality, planned items modularity, work packages traceability and plan items reestimation will be measured and combined. Applied the quantification mechanism of WBSPCM model, WBS-based plan changeability problems and defects can be easily identified. Used rule-based correction and improvement manner can concretely increase WBS-based plan changeability and reduce software development risk. In Section II, survey the relationship between the SPMP and WBS-based plan, and describe critical factors of software project failure. In Section III, changeability factors which affect WBS-based plan revision and adjustment capability are listed and discussed. In Section IV, propose a WBSPCM model, and present a rule-based changeability defects identification and improvement manner. In Section V, collect advantages of WBSPCM model and make a conclusion for the paper.

\section{WBS IMPORTANCE AND SOFTWARE PROJECT FAILURE FACTORS}

\section{A. The Relationship between SPMP and WBS}

There are many change challenges will affect the operations of software project. How to reduce the impact of 
change events becomes a critical issue to reduce development risk. According to the client provided cost budget, schedule, resource, functional and non-functional requirements, the SPMP must defines software development steps, work package schedule, organization architecture and resource planning. Initial version of the SPMP is drawn up and released when the specifications have been signed off by the client. Based on the SPMP, software development operation executes with developing schedule, planning works, and meeting each requirement items which proposed by the client. In order to concretely and effectively accomplish requirement specifications, large scale and complicate project should be suitable decomposed. For simple and clear work items, it is easy to define schedule, budget, organization hierarchical and resource allocation etc. plans. WBS is a critical item for decomposing complicate and large scale software project. WBS is a product-oriented method and uses hierarchical diagram for work items [5]. Based on WBS, software system can be well layered and decomposed into high manageable work items. For simple and manageable work items, it is easy to define schedule, budget, applied tool and technology, resource allocation and risk control etc. plans. Decomposed work items of WBS should have clear mission, concrete range, priority relationship with others items, reference relationship with others items, and easy elicited estimation parameters etc. features, description as follows (show as Fig. 1).

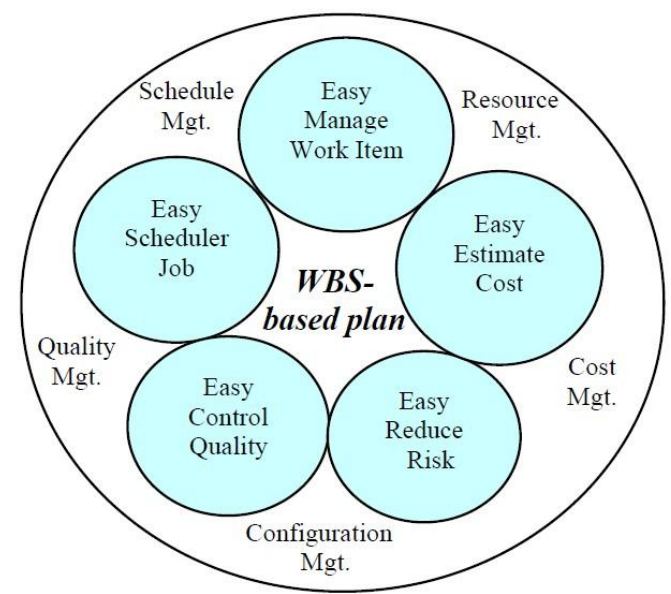

Fig. 1. Five major features of WBS-based plan.

- Decomposing manageable work items: Multi-layer decomposition makes software system with more manageable work items.

- Easy schedule work items: Decomposed work item has clear task and objective. For low layer item or high layer package easy assign task time and priority, and assist resource management and schedule management planning.

- Easy control work items quality: simple work item easy collect operation data and quality factors. The information can help supervise and manage operation efficiency and quality of work item.

- Easy estimate work items cost: there are several cost estimation models such as function point [7], COCOMO [8], expert adjustment, and analogy methods. No matter which one model, simple and clear mission and objective always can increase estimation accuracy. In addition, estimation cost can easy transform into others planning items.

- Easy reduce work items risk: problems or defects which occurred in work item, can easy isolate and revise to reducing development risk.

Finally, WBS-based plan work items are integrated by bottom up. One time one layer, work items are combined into budget, schedule, quality, configuration, and resource management plans. Based on WBS, each management plans can overcome any change events and immediately accomplish plan modification and adjustment. The relationship between SPMP and WBS is shown as Fig. 2 .

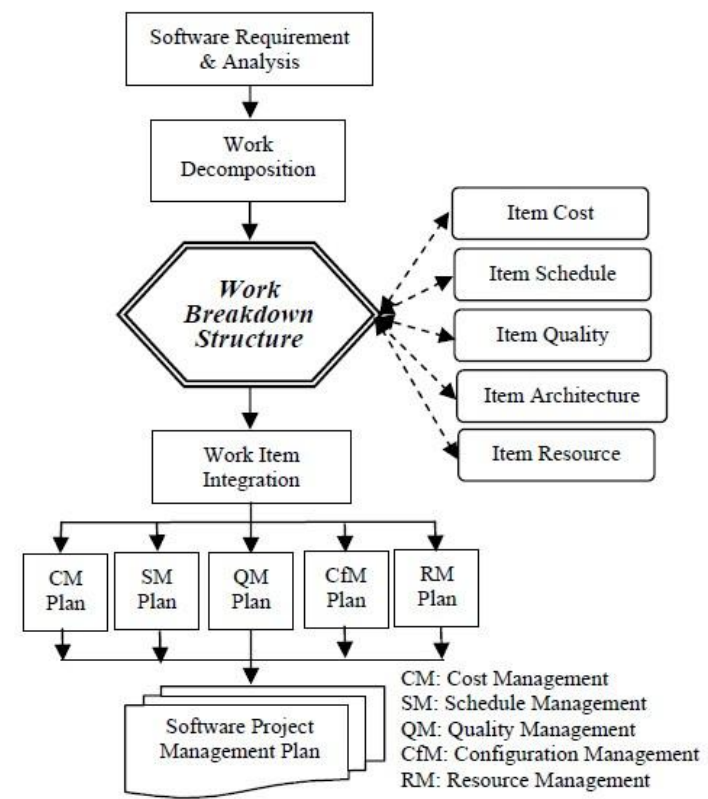

Fig. 2. Relationship between WBS and SPMP

\section{B. Critical Factors of Software Project Failure}

According to the Standish group study report which investigated large volume software project, the success rate of software project only approach one third [9], [10]. And, $80 \%$ failure software projects suffer from some troubled problems which include schedule delay, insufficient budget and incomplete requirements. High risk of software project comes from the situations of schedule delay, insufficient budget and unfinished requirements [11]-[13]. Four critical change events are major reason to cause the software project with high risk, describe as follows:

1) Initial phase of software development, system analyst collect incomplete information and documents to cause to system requirement specification and user requirement have differences.

2) In software development process, new technologies and operation environment are innovated continuously. It causes the original plans can not completely match with new technologies and operation environment.

3) In requirement phase, the client proposed many system requirements. However, in software development process, parts of the requirement items will be required to adjust, modify or delete. Even some new requirements are required to append into the system. These change requests will greatly impact to follow up software development operations.

4) Each phase operation of software project needs different resource which includes developers, hardware devices, 
software tools and development environment. Resource allocation may happen to personnel movement, facility delayed delivery, hardware breakdown, equipment failure and obsolete devices. These resource change events may increase software development risk.

Summary the above description, incomplete system requirements, technology and environment evolution, client change requests and resource reallocation are four major change events (shown as in Fig. 3).

In software development process, these change events can not be avoided or excluded, will be cause different level impacts to project planned items. Change events are also a critical reason of software project failure. Therefore, for reducing the failure nightmare, the WBS-based plan should have high changeability to handle each kinds change events. It is because that new version of the SPMP must be released continually until project finish or terminal.

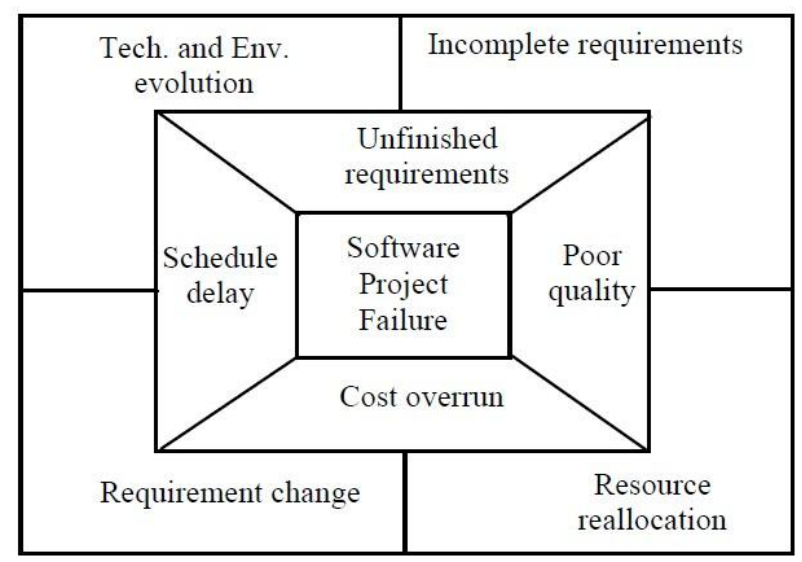

Fig. 3. Four change events and their impacts.

\section{Changeability FACTORS OF WBS-BASEd Plan}

WBS-based plan is the major basis for software project operation and development. However, unexpected change events always cause that WBS-based plan has to revise and adjust. A major reason of project failure is WBS-based plan cannot revise and adjust in time after change events occurred. High changeable quality plan can collate change claims to revise and adjust the planned items for handling each kinds change events. Enhancing WBS-based plan changeability is critical method to reduce project failure risks. According to McCall's quality factor model description [14] and ISO 9126 quality architecture [15], software quality factors and characteristics have precise hierarchical structure. In ISO 9126, changeability is a major indicator for software quality. However, software changeability is generally focused on development document and source code, but not focused on work items. Correctness, completeness, consistency and readability four basic document qualities make the software plan to have flexible revision and adjustment. In design phase, modularity is a necessary characteristic to increase module dependence and maintainability. Software work items also need modularity to increase WBS-based plan changeability [16]. Traceability is a critical characteristic to identify cross relationship between work items. Based on traceability, the work items affected by change events can be isolated quickly and completely. For improving WBS-based plan changeability, basic document quality, modularity, traceability and re-estimation capability four quality characteristics are discussed as follows:

1) Basic document quality: Quality of work item is an important basis for revising or adjusting planned document. Four major quality characteristics of planned document, include correctness, completeness, consistency and readability, describe as follows:

- Correctness: Incorrect planned documents may affect the results of work items revision and adjustment.

- Completeness: Incomplete work item documents may cause more defects and errors after software project revision and adjustment.

- Consistency: Inconsistent work item documents may cause more defects and errors after software project revision and adjustment.

- Readability: Unreadable work item documents may cause more defects and errors after software project revision and adjustment.

2) Modularity: After work breakdown, each work packages have assigned specific job for achieving user requirements. These work packages should own high modularity to handle change events. There are three major factors to affect modularity.

- Coupling: For increasing independence, work items should have as low coupling as possible with others work items.

- Cohesion: For increasing independency, internal subtasks of work items should have as high cohesion as possible.

- Complexity: Work items with high complexity may reduce change flexibility for each change events.

3) Traceability: WBS-based plan with high traceability can help identify the affected work packages of plan changes. Internal and external cross-reference table (CRT) of work packages are two major factors to affect the traceability of WBS-based plan.

- Internal CRT: Internal CRT of work package can easy identify the task inter-relationships in work item.

- External CRT: External CRT of work package can easy identify the work item inter-relationships after system break down.

4) Re-estimation: After plan changes, the budget, schedule, quality and resource of affected items should re-estimate. Clear estimation method [7], [8] can efficiently increase accurate re-estimation.

- Parameters: The parameter data for project effort estimation should have clear definition and easy acquisition. Referring to the clear parameter data, estimation formula can effectively handle reestimation.

- Formula: Combining simple with accurate estimation formula, the revised work item can quick and accurately re-estimate development effort.

- Transformation: Re-estimated software development effort is just an item of development expenses. Development effort needs to transform to other items of development explain. Simple and clear transformation method can easily transfer software development effort into budget, schedule and resource expenses. 


\section{Changeability Measurement Model}

\section{A. Changeability Factor Collection}

In system analysis phase, the drafted requirement specification must pass the client confirmation and become the formal requirement specification [3], [16]. According to the formal requirement specification, the SPMP should be completely developed and clearly defined. For reducing the complexity of software project, WBS should be considered for project planning. In WBS-based plan, complex software planning and development missions are decomposed into several levels. Work packages and tasks become the critical planned items. After completion of task planning, next step must conduct a formal task plan review activity to identify planned defects and collect task changeability factors. Task is part of work package. Task planned items are combined into a work package plan. After completion of work package planning, next step must conduct a formal work package review activity to identify planned defects and collect work package changeability factors. Work package is part of software project. Work package plans are combined into over software project plan. Based on IEEE 1058 SPMP format, software project plan is written down. After completion of SPMP, following step must conduct a formal WBS-based plan review activity to identify planned defects and collect WBS-based plan changeability factors. Clear inspection checked items can effectively increase the review activities performance. So, it is necessary to develop a set of complete and clear inspection checklists for conducting review activity. The mission of checklist formulation should be delivered to senior software engineer and the experience wealth software project management experts. Review activity with inspection checklist can collect the complete WBSbased plan changeability factors. Collecting changeability factors are critical operation for quantifying the characteristics of changeability.

\section{B. Changeability Measurement Model}

Individual factor or measurement can only measure or evaluate the specific quality characteristic. In order to effectively monitor and assess the changeability problem and defect, individual measurements should make the appropriate combination [6], [8], [14], [18], [19]. Two kind of metric combination models are Linear Combination Model (LCM for short) [6], [14], [18] and Non-Linear Combination Model (NLCM for short) [8], [14], [19]. NLCM has higher accuracy measurement than LCM. However, LCM has high flexibility, more extensible and easy formulation than NLCM. For this, in this paper, LCM is applied to changeability measurement.

The different level planning activities have different quality metrics be shown. Therefore, before applying the linear combination model, the quality factors must be normalized. Refer to predefined weight values and four combination formulas, basic level quality factors can be combined into the four quality characteristic measurements. Finally, the formula combines with four quality characteristic measurements into a SPMP changeability indicator. Five formulas described as follows:

1) Basic Document Quality Measurement (BDQM) is combined with correctness, completeness, consistency, and readability four characteristics. The combination formula is shown as Equation (1):
BDQM: Basic Document Quality Measurement

$$
\begin{array}{cc}
\text { CrF : Correctness Factor } & W_{c r f}: \text { Weight of CrF } \\
\text { CmF : Completeness Factor } & W_{c m f}: \text { Weight of CmF } \\
C n F: \text { Consistency Factor } & W_{c n f}: \text { Weight of CnF } \\
\text { RF : Readability Factor } & W_{r f}: \text { Weight of RF }
\end{array}
$$

$$
\begin{gathered}
B D Q M=W_{c r f} * C_{r}+W_{c m f} * C_{m} F+W_{c n f} * C_{n} F+W_{r f} * R F \\
W_{c r f}+W_{c m f}+W_{c n f}+W_{r f}=1
\end{gathered}
$$

2) Work Item Modularity Measurement (WIMM) is combined with coupling, cohesion and complexity three factors. The formula is shown as Equation (2):

WIMM: Work Item Modularity Measurement

$$
\begin{array}{ll}
C U: \text { Coupling Factor } & W_{c u}: \text { Weight of } C U \\
C O: \text { Cohesion Factor } & W_{c o}: \text { Weight of } C O \\
C X: \text { Complexity Factor } & W_{c x}: \text { Weight of } C X
\end{array}
$$

$$
\begin{aligned}
& \text { WIMM }=W_{c u} * C U+W_{c o} * C O+W_{c x} * C X \\
& W_{c u}+W_{c o}+W_{c x}=1
\end{aligned}
$$

3) Work Item Traceability Measurement (WITM) is combined with internal cross-reference table and external cross-reference table two factors. The combination formula is shown as Equation (3):

WITM: Planned Item Traceability Measurement

$$
\begin{array}{ll}
\text { ICRT: Internal CRT } & W_{\text {icrt: }}: \text { Weight of ICRT } \\
\text { ECRT: External CRT } & W_{\text {ecrt }}: \text { Weight of ECRT }
\end{array}
$$

$W I T M=W_{i c r t} * I C R T+W_{\text {ecrt }} * E C R T \cdots W_{i c r t}+W_{\text {ecrt }}=1$

4) Work Package Re-estimation Measurement (WPRM) is combined with coupling metric, parameter consistency, and cross reference etc. three factors. The combination formula is shown as Equation (4):

WPRM: Work Package Re-estimation Measurement

$\begin{array}{ll}P F: \text { Parameter Factor } & W_{p f}: \text { Weight of PF } \\ F F: \text { Formula Factor } & W_{f f}: \text { Weight of FF } \\ \text { TR: Transformation Factor } & W_{t r}: \text { Weight of TR }\end{array}$

$$
\begin{aligned}
& W P R M=W_{p f} * P F+W_{f f} * F F+W_{t r} * T R \\
& W_{p f}+W_{f f}+W_{t r}=1
\end{aligned}
$$

Finally, combine BDQM, WIMM, WITM and WPRM quality measurements into a WBS-based Plan Changeability Quality Indicator (WPCQI). The formula is shown as Equation (5).

WPCQI: WBS-based Plan Changeability Quality Indicator

BDQM: Basic Document Quality Measurement

$$
W_{q m} \text { : Weight of } B D Q M
$$

WIMM: Work Item Modularity Measurement

$$
W_{m m} \text { : Weight of PIMM }
$$

WITM: Work Item Traceability Measurement 


$$
W_{t m}: \text { Weight of PITM }
$$

WPRM: Work Package Re-estimation Measurement $W_{r m}$ : Weight of WPRM

$W P M Q I=W_{q m} * B D Q M+W_{m m} * W I M M+W_{t m} * W I T M+W_{r m} * W P R M$ $W_{q m}+W_{m m}+W_{t m}+W_{r m}=1$

In WBS-based plan inspection activity, the factors which affect change operation were collected and divided into 12 groups. In first layer, 12 groups quality factor are combined into 12 quality factor metrics. In second layer, 12 quality factor metrics are combined into four quality characteristic measurements. In third layer, four quality characteristic measurements are combined into WPMQI. With three layer combination formulas to generate WPMQI, the process is called a WBS-based Plan Changeability Measurement (WBSPCM) model. The WBSPCM model architecture is shown as Fig. 4.

\section{Rule-Based Plan Changeability Improvement Manner}

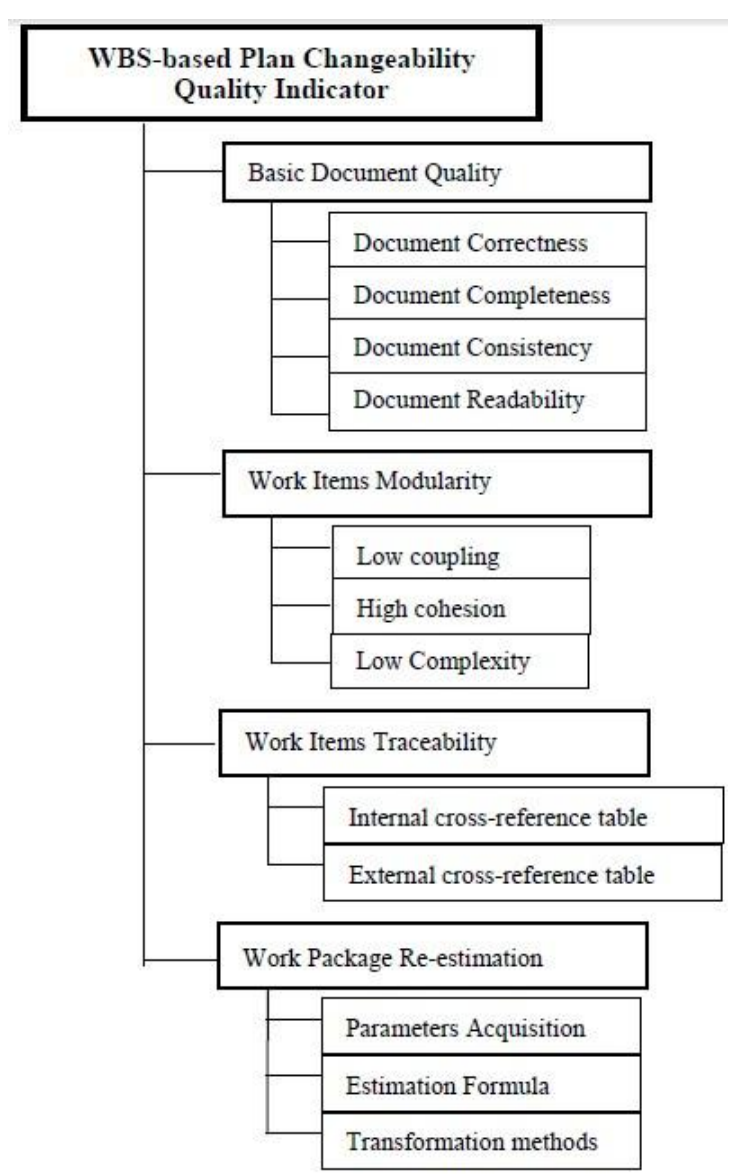

Fig. 4. The architecture of WBSPCM model.

WPCQI is a relative judgment mechanism also is a basis to identify problem or defect of WBS-based plan changeability. In WBSPCM model, basic quality factors are combined into high level measurement. High level characteristic measurements are combined into a WPMQI. Therefore, if the WPCQI does not satisfy quality criterion, it represents WBS-based plan has changeability defect. Based on the combination formulas, high level characteristic measurement mapping to the planning tasks should be rigorously inspected to identify the problem or defect and propose the corrective action. This paper proposed the rulebased WBS changeability improvement activity for corrective action, described as follows:

IF measurement not satisfy "quality criterion"

THEN identify the problem and defect, and correct it.

\section{CONCLUSION}

Success software project must conquer plan change challenges. The change events, which include error correction, requirement revision, environment evolution, and resource adjustment, may cause to plan change. Any plan change always affects to software development process and increase software development risk. Plan change not only has to invest extra resource and cost, but also may cause project delay. Handling plan changes, the affected development documents and tasks can not be effectively isolated will increase software development risk. In addition, the affected development documents and plan items can not be concretely and correctly modified will greatly reduce project success ratio. For this, WBS-based plan should have high changeability to reduce plan change risk. For improving changeability of WBS-based plan, the paper proposed a WBS-based Plan Changeability Measurement (WBSPCM) model. WBS-based plan with high changeability can enhance plan items isolation capability and modification flexibility to reduce development risk. The advantages of WBSPCM model are described as follows:

1) Identify WBS-based plan changeability defects and provide revision suggestions.

2) Improve WBS-based plan changeability continuously.

3) Combination formula of WBSPCM model has precise, simple and flexible adjustment features.

\section{ACKNOWLEDGMENT}

The paper is sponsored by Shih Chien University 2012 Management College research funding.

\section{REFERENCES}

[1] K. Mohan, P. Xu, and B. Ramesh, "Improving the changemanagement process," CACM, vol. 51, no. 5, pp. 27-34, 2008.

[2] D. Litten. (Oct. 2009). Using Change Management and Change Control Within a Project. [Online]. Available: http://www.projectsmart.co.uk/pdf/using-change-management-andchange-control-within-a-project.pdf

[3] S. R. Schach, Object-Oriented Software Engineering, McGraw-Hill Companies, 2008

[4] IEEE std. 1058-1998, IEEE Standard for Software Project Management Plans, IEEE, New York, 1998.

[5] Jr. S. J. Mantel, J. R. Meredith, S. M. Shafer, and M. M. Sutton, Project Management in Practice, 4th edition, John Wiley \& Sons, Inc., 2011.

[6] S. T. Lai and C. C. Yang, "A software metric combination model for software reuse," in Proc. 1998 Asia-Pacific Software Engineering Conference, Taiwan, Taipei, pp. 70-77, 1998

[7] G. C. Low and D. R. Jeffrey, "Function points in estimation and evaluation of the software process," IEEE Trans. On Software Engineering, vol. 16, no. 1, pp. 64-71, 1990.

[8] B. W. Boehm, Software Engineering Economics, Prentice-Hall, New Jersey, 1981.

[9] J. L. Eveleens and C. Verhoef, "The rise and fall of the chaos report figures," IEEE Software, vol. 27, no. 1, pp. 30-36, 2010.

[10] The Standish group. (April 23, 2009). New Standish Group report shows more project failing and less successful projects. [Online]. Available: http://www.standishgroup.com/newsroom/chaos_2009.php

[11] B. W. Boehm, "Software risk management: Principles and practices," IEEE Software, vol. 8, no. 1, pp. 32-41, 1991 
[12] B. W. Boehm, Tutorial: Software Risk Management, Los Alamitos, Calif: IEEE CS Press, 1989.

[13] R. Fairley, "Risk management for software projects," IEEE Software, vol. 11 , no. 3, pp. 57-67, 1994

[14] D. Galin, Software Quality Assurance-From theory to implementation, Pearson Education Limited, England, 2004.

[15] ISO/IEC: FCD 9126-1.2: Information Technology-Software Product Quality, Part I: Quality Model, 1988.

[16] E. F. Weller, "Using metrics to manage software projects," IEEE Computer, vol. 27, no. 9, pp.27-24, 1994.

[17] R. S. Pressman, Software Engineering: A Practitioner's Approach, New York: McGraw-Hill, 2010.

[18] S. D. Conte, H. E. Dunsmore, and V. Y. Shen, Software Engineering Metrics and Models, Benjamin/Cummings, Menlo Park, 1986.
[19] N. E. Fenton, Software Metrics-A Rigorous Approach, Chapman \& Hall, 1991.

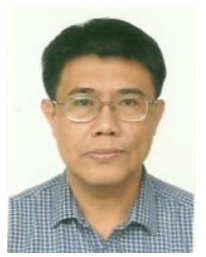

Sen-Tarng Lai was born in Taiwan in 1959. He received his BS from Soochow University, Taiwan in 1982, master from National Chiao Tung University, Taiwan in 1984 and $\mathrm{PhD}$ from National Taiwan University of Science and Technology, Taiwan in 1997. His research interests include software security, software project management, and software quality. He is currently an assistant professor in the Department of Information Technology and Management at Shin Chien University, Taipei, Taiwan. 